\title{
Myths and misperceptions in pacemaker patients: a quasi experimental study to assess effect of an educational intervention
}

\author{
Ghulam Kubra, Fariha Hasan, Faisal Qadir, Shazia Rasheed and Azam Shafquat* [D
}

\begin{abstract}
Background: Misconceptions regarding activities after pacemaker implantation can result in restrictions in daily life. This study aims at measuring the correction of misconceptions following an educational intervention utilizing a picture based brochure and personal counseling.

Methods: One hundred and twenty-eight patients were enrolled in a quasi experimental study on the day after pacemaker implantation. Patients' perceptions about safety of various daily activities, medical procedures, and usage of appliances, in the presence of pacemaker was assessed using a questionnaire before and ten days after an educational intervention using a pictorial brochure. The number of correct responses before and after the intervention was compared to assess the change in patients' perception.

Results: A total of 128 patients were interviewed at baseline, of which 115 followed-up at 10 days. Mean age of patients was $60.31 \pm 12.81$ years. In total, 76 (59.4\%) were male and 61 (47.7\%) were illiterate. Mean correct responses increased from $64.47 \pm 29.48 \%$ to $92.29 \pm 14.42 \%$ ( $p<0.001$ ). Percent of questions answered correctly improved in all three categories after the intervention. Correct answers increased from $74.57 \pm 24.94 \%$ to $95.65 \pm 11.48 \%(p<0.001)$ for routine daily activities, $51.09 \pm 33.9 \%$ to $84.78 \pm 20.86 \%$ ( $p<0.001$ ) for medical procedures and $64.03 \pm 37.36 \%$ to $92.57 \pm 18.79 \%(p<0.001)$ for domestic appliance usage.
\end{abstract}

Conclusion: Misconception that can adversely affect pacemaker patient's quality of life can be corrected by counseling with pictorial based brochures regardless of the patient's baseline knowledge or education.

Keywords: Cardiac pacemaker, Artificial, Education, Patient, Electric power supplies/adverse effects, Electromagnetic fields/adverse effects, Household articles/instrumentation

\section{Background and objective}

Pacemaker implantation for bradycardia can alleviate symptoms and possibly arrhythmia related death [1]. Post-pacemaker implantation patients should be able to resume most routine activities. However, studies have shown that patients may practice self-imposed restrictions that can affect their quality of life [2].

*Correspondence: azamshafquat@gmail.com

National Institute of Cardiovascular Diseases, Rafiqui Shaheed Road, Karachi 75510, Pakistan
Common misbeliefs relate to posture and activity (e.g.: not sleeping on the side of pacemaker, restricting arm movements, not climbing stairs), avoiding use of common house hold appliances (including electrical switches, mobile phones, television, gas/microwave ovens) and restriction of various medical procedures (X-ray exposure, ultrasound, CT (computerized tomography) scan [3]

There is limited data on pacemaker patients' postimplant life style from developing countries which may have a high proportion of illiterate population [4]. Full benefit of expensive therapies in resource limited 
environments may not be achieved without adequate patient teaching. We hypothesized that a visual-assisted educational intervention would significantly improve the patient's knowledge of permissible activities after pacemaker implantation and would persist at short-term follow-up.

The objective of this study was to measure pacemaker patient's knowledge of permissible activities before and after an educational intervention.

\section{Methods}

\section{Patients}

Patients with new pacemaker implantation were included in this single center study at National Institute of Cardiovascular Diseases (NICVD) Karachi, Pakistan. NICVD is a publicly funded tertiary single specialty cardiac institute comprising of nine hospitals which caters to all socioeconomic sections of the population as no payment is required of the patients. Consenting adult (over 18 years of age) inpatients were enrolled on the day following pacemaker implant. Patients who had communication disabilities or unable to speak one of the local languages were excluded.

\section{Study design and sample calculation}

A quasi experimental design was used. Sample size estimation was based on patients' knowledge regarding safe activities from prior study [3]. Estimating knowledge retention of $75 \%$ after ten days, at $95 \%$ confidence level and $80 \%$ power of test and with $20 \%$ of difference as clinical significance value, a sample size of eighty-five patients was calculated. With an expected loss to follow-up of $50 \%, 128$ patients were enrolled.

\section{Knowledge assessment and educational intervention}

On post-operative day one after pacemaker implantation a previously validated questionnaire [3] was administered. Patient's knowledge regarding use of eleven electronic appliances (such as use of mobile phones, electrical switches, iron and operating electric motors), eight daily activities (such as driving, climbing stairs, moving and sleeping on the side of pacemaker), and four medical procedures (X-ray, CT Scan and MRI (magnetic resonance imaging)) were obtained before educational intervention. This was followed by an educational intervention by verbal counseling in the patient's language using a pictorial brochure (Fig. 1). Where possible the patients were encouraged to do these activities in front of the counselor. The questionnaire was re-administered on first routine follow-up visit at ten days (Fig. 2). Pre- and post-intervention percent total correct response score was computed as 100*(number of correct responses)/ total attributes for each patient.

\section{Statistical analysis}

SPSS version 21 was used to analyze the collected data. Data were represented as frequency (\%) or mean \pm standard deviation. Pre- and post-intervention responses on categorical response variables were compared by applying McNemar test, and for continuous response variables appropriate paired sample $t$ test or Wilcoxon signed-rank test was applied. Criteria for statistical significance will be taken as $p$-value of less than or equal to 0.05 .

\section{Results \\ Patient characteristics}

Of the 128 patients who had the initial questionnaire administered, 13 did not return for day 10 follow-up visit and 115 patients were included in the post-intervention analysis. (Fig. 1).

Seventy-six (59.4\%) patients were male. Mean age was $60.31 \pm 12.81$ years and $61(47.7 \%)$ of baseline patients were illiterate (unable to read or write any language). The patient's household size on the average was $7.61 \pm 4.61$ and $87(67.9 \%)$ of patients had a monthly household income less than 30,000 Pakistani rupees (equivalent to178.52 USD) (Table 1).

\section{Perception of activities before intervention}

Mean correct responses for 128 patients at baseline was $64.47 \pm 29.48 \%$ (Table 2). Percent of questions answered correctly was $74.57 \pm 24.94 \%$ for routine daily activities, $51.09 \pm 33.9 \%$ regarding medical procedures and $64.03 \pm 37.36$ regarding safe use of domestic appliance.

Fifty-six (43.7\%) patients felt that electric wall switches and outlets are unsafe for pacemaker patients to use and $58(45.3 \%)$ patients advised against using an electric iron. Similar misconception for usage of other house hold appliance like microwave ovens and UPS (Uninterrupted Power Supply) and electricity generators were noted in 58(45.3\%) and 61(47.7\%) patients, respectively. Fiftyone $(40 \%)$ of the patients stated that pacemaker patients should not sleep on the side of the implanted device. About $60 \%$ felt that mobile phones should not be used. Similar misconception was reported for other daily routine activities and medical appliances. (Table 2).

\section{Perception of activities after intervention:}

In the repeat assessment 10 days after the education intervention the patient's perception of activities and appliance usage changed. Mean correct responses increased from $64.47 \pm 29.48 \%$ to $92.29 \pm 14.42 \%$. Percent of questions answered correctly improved in all three categories after the intervention. Correct answers increased from $74.57 \pm 24.94 \%$ to $95.65 \pm 11.48 \%$ 


\section{INFORMATION FOR PATIENTS WITH PERMANENT PACEMAKER}

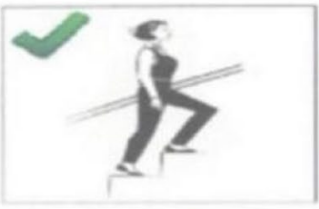

You can climb stairs

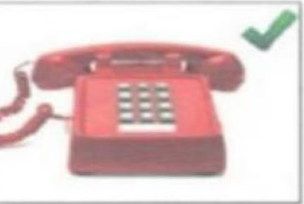

You can use a landline phone

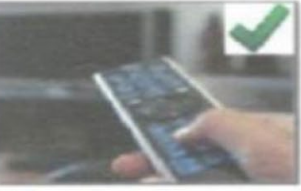

You can use remote control

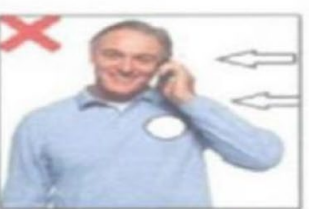

Don't use mobile phone on same side as pacemaker

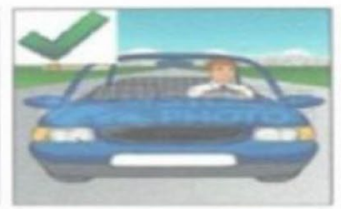

You can travel in and drive an automobile

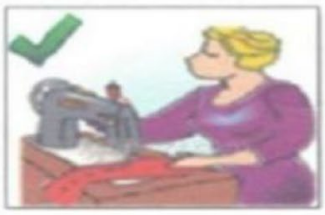

You can use sewing machine

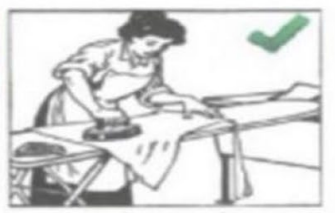

You can use iron and other household appliances

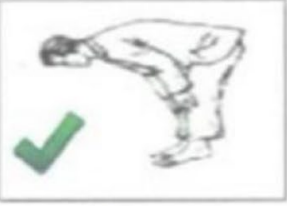

You can bend over

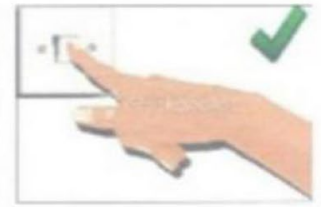

You can use electrical switches

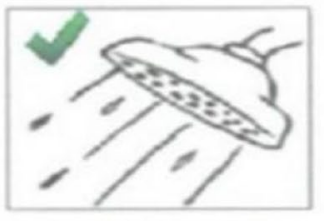

You can shower and bath after your wound heals

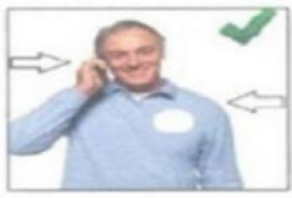

You can use a mobile phone on ear opposite to the pacemaker side

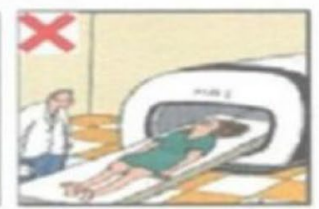

Do not get an MRI done without informing your doctor

Fig. 1 Educational brochure

$(p<0.001)$ for routine daily activities, $51.09 \pm 33.9 \%$ to $84.78 \pm 20.86 \%(p<0.001)$ for medical procedures and $64.03 \pm 37.36 \%$ to $92.57 \pm 18.79 \%(p<0.001)$ for domestic appliance usage.

After the intervention significantly more patients felt using electrical switches to turn on lights 111 (96.5\%) and using an electric iron 109 (94.8\%) were safe activities ( $p<0.001$ compared to before). Similarly 111(96.5\%) of patients felt it was safe to use mobile phones. Significant improvement in correct perceptions was seen across all activities that were assessed (Table 2).

\section{Subgroup analysis of changes in perception}

At baseline, men had significantly more correct responses $70.08 \pm 27.48 \%$ compared to women with $56.27 \pm 30.64 \%$. Post-intervention they both improved significantly with men getting $92.94 \pm 13.57$ and women $91.3 \pm 15.72$ correct responses. After the intervention, there was no longer any significant difference between the genders $(p=0.565)$ (Table 3).

Similarly, illiterate patients had significantly lower correct responses, $53.88 \pm 29.55$ compared to literate patients $74.11 \pm 26.09$. After the intervention, there was 


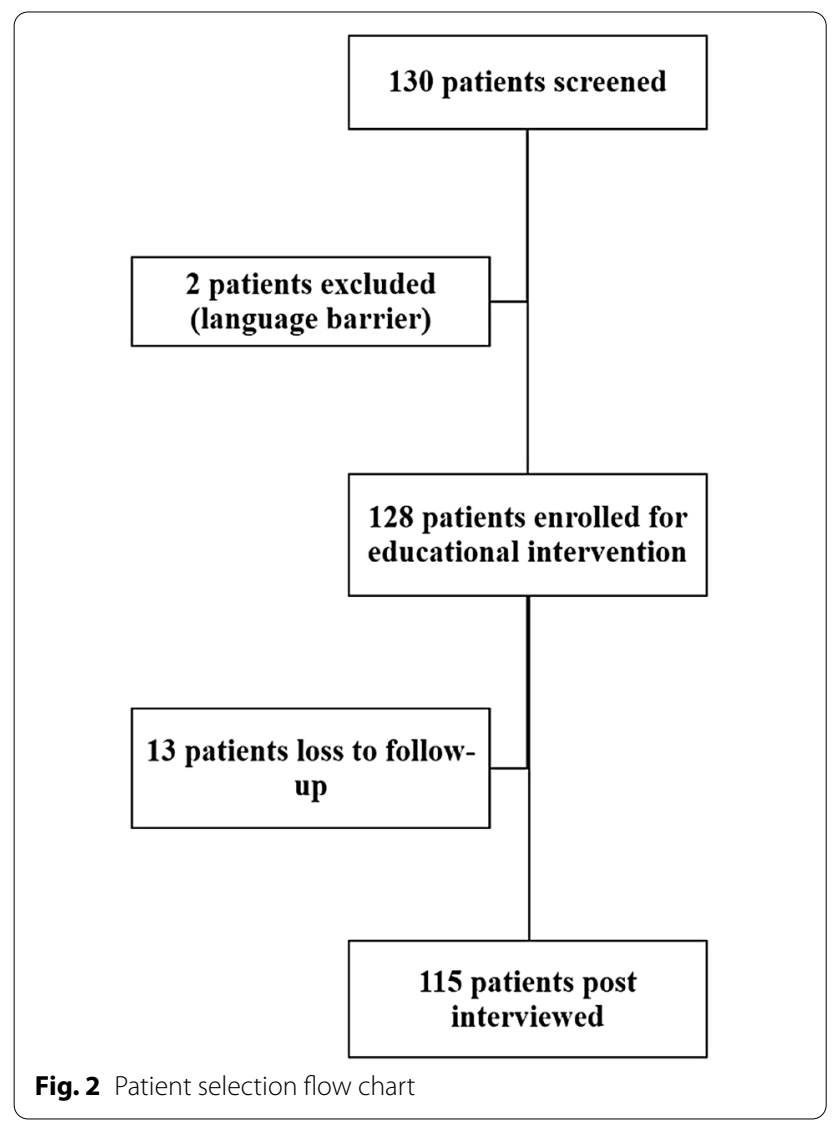

Table 1 Baseline demographic characteristics

\begin{tabular}{ll}
\hline Characteristics & Total \\
\hline Total (N) & 128 \\
Gender & \\
Male & $76(59.4 \%)$ \\
Female & $52(40.6 \%)$ \\
Age (years) & $60.31 \pm 12.81$ \\
Qualification & \\
Illiterate & $61(47.7 \%)$ \\
Can read and write & $12(9.4 \%)$ \\
Primary level (up to 6th grade) & $20(15.6 \%)$ \\
Secondary level (up to 10th grade) & $12(9.4 \%)$ \\
Intermediate level (up to 12th grade) & $11(8.6 \%)$ \\
Higher education (> 12th grade) & $12(9.4 \%)$ \\
Household (HH) size & $7.61 \pm 4.61$ \\
Monthly HH income (Pakistani Rupees) & \\
$<15,000$ & $51(39.8 \%)$ \\
15,000-30,000 & $36(28.1 \%)$ \\
30,000-45,000 & $13(10.2 \%)$ \\
$45,000-60,000$ & $6(4.7 \%)$ \\
$>60,000$ & $22(17.2 \%)$ \\
\hline
\end{tabular}

no statistical difference between the two groups as they both had more than $90 \%$ correct responses (Table 3 ).

\section{Discussion}

This study adds to the limited literature from developing countries related to misconceptions that can disrupt routine activities after pacemaker implantation. We report a marked improvement in patients' knowledge persisting for at least ten (10) days after an educational intervention.

Our main findings showed that the majority of patients had incorrect perceptions pertaining to one or more common daily activity. About half of the respondents felt patients with pacemakers should not sleep on side of implanted pacemaker, touch an electrical switch, use a mobile phone, drive a car, or turn on a domestic electric appliance like ovens, UPS and electric generators. However, it was very encouraging that a simple intervention using a pictorial brochure brought changes in knowledge that lasted till their first follow-up visit.

Although, pacemaker implantation can provide symptomatic relief, uncertainty in daily routine activities can be a source of distress for them. Fear of unemployment, feeling of inadequacy at work place especially while carrying out physical work are real life experiences of the patient which are often groundless [5]. In a South African study up to half of the patients experienced handicapped life post-device implantation and felt they had restricted activities after the device compared to before [2]. An earlier study from our institution reported similar results with a third of the patients feeling they should restrict many daily activities, and more than half felt unsafe with usage of electrical and other household appliances [3]. Similar to these studies our current study shows that between half to two thirds of the participants incorrectly perceive many routine activities as inappropriate for patients with pacemakers.

Patients' socioeconomic status including affluence, educational background as well as their bio psycho social profile greatly influences their adjustment to life after pacemaker implantation [6]. In our study, the knowledge level at baseline was not significantly different between genders or based on income. However, illiterate patients were significantly poorer in knowledge at baseline. It is extremely gratifying that when a simple educational intervention was performed in these patients, they came at par with those with education.

Detailed counseling sessions by a trained professional have been shown to be helpful [6]. However, our study shows that a simple intervention by a member of the pacemaker implantation team can bring significant changes regardless of the patients underlying demographics. 
Table 2 Number (\%) of patients correctly answering questions on permissible activities pre- and post-intervention

\begin{tabular}{|c|c|c|c|}
\hline \multirow[t]{2}{*}{ Characteristics } & \multicolumn{2}{|l|}{ Intervention } & \multirow[t]{2}{*}{ P-value } \\
\hline & Pre & Post & \\
\hline Total (N) & 128 & 115 & - \\
\hline \multicolumn{4}{|l|}{ Various daily activity } \\
\hline Travel in a car & $105(82 \%)$ & 114 (99.1\%) & $<0.001^{*}$ \\
\hline Drive a car & $66(51.6 \%)$ & 107 (93\%) & $<0.001^{*}$ \\
\hline Bath and swim & $108(84.4 \%)$ & $112(97.4 \%)$ & $<0.001^{*}$ \\
\hline Climb stairs & $96(75 \%)$ & $112(97.4 \%)$ & $<0.001^{*}$ \\
\hline Prayers (bend over, kneel forward) & $103(80.5 \%)$ & $112(97.4 \%)$ & $<0.001^{*}$ \\
\hline Not passing through metal detectors & $92(71.9 \%)$ & $105(91.3 \%)$ & $<0.001^{*}$ \\
\hline Sleep on side of pacemaker & $77(60.2 \%)$ & 105 (91.3\%) & $<0.001^{*}$ \\
\hline Move arm on side of pacemaker & $100(78.1 \%)$ & $113(98.3 \%)$ & $<0.001^{*}$ \\
\hline Mean correct response rate & $74.57 \pm 24.94 \%$ & $95.65 \pm 11.48 \%$ & $<0.001^{*}$ \\
\hline \multicolumn{4}{|l|}{ Medical procedure } \\
\hline X-ray & $87(68 \%)$ & $111(96.5 \%)$ & $<0.001^{*}$ \\
\hline CT Scans & $53(41.4 \%)$ & $74(64.3 \%)$ & $<0.001^{*}$ \\
\hline MRI should not be done & $44(34.4 \%)$ & $97(84.3 \%)$ & $<0.001^{*}$ \\
\hline Ultrasound & $84(65.6 \%)$ & $108(93.9 \%)$ & $<0.001^{*}$ \\
\hline Mean correct response rate & $51.09 \pm 33.99 \%$ & $84.78 \pm 20.86 \%$ & $<0.001^{*}$ \\
\hline \multicolumn{4}{|l|}{ Usage of appliances } \\
\hline Mobile phones & $85(66.4 \%)$ & $111(96.5 \%)$ & $<0.001^{*}$ \\
\hline Standard telephones & $91(71.1 \%)$ & $110(95.7 \%)$ & $<0.001^{*}$ \\
\hline Irons & $70(54.7 \%)$ & 109 (94.8\%) & $<0.001^{*}$ \\
\hline Touch electrical switches & $72(56.3 \%)$ & $111(96.5 \%)$ & $<0.001^{*}$ \\
\hline Microwave ovens & $70(54.7 \%)$ & $97(84.3 \%)$ & $<0.001^{*}$ \\
\hline TV, VCR, Radios & $89(69.5 \%)$ & $108(93.9 \%)$ & $<0.001^{*}$ \\
\hline Remote controls & $91(71.1 \%)$ & 108 (93.9\%) & $<0.001^{*}$ \\
\hline Electric sewing machines & $75(58.6 \%)$ & 107 (93\%) & $<0.001^{*}$ \\
\hline Gas oven & $92(71.9 \%)$ & $104(90.4 \%)$ & $<0.001^{*}$ \\
\hline UPS and electric generators & $67(52.3 \%)$ & $101(87.8 \%)$ & $<0.001^{*}$ \\
\hline Electrical Motors (for pumping water) & $81(63.3 \%)$ & $105(91.3 \%)$ & $<0.001^{*}$ \\
\hline Mean correct response rate & $64.03 \pm 37.36 \%$ & $92.57 \pm 18.79 \%$ & $<0.001^{*}$ \\
\hline Total mean correct response rate & $64.47 \pm 29.48 \%$ & $92.29 \pm 14.42 \%$ & $<0.001^{*}$ \\
\hline
\end{tabular}

${ }^{*}$ significant at $5 \%$

CT scan: computerized tomography, MRI: magnetic resonance imaging, TV: television

Table 3 Pre- and post-intervention mean correct response rate stratified by gender, literacy and income

\begin{tabular}{|c|c|c|c|c|c|}
\hline \multirow[t]{2}{*}{ Characteristics } & \multicolumn{2}{|c|}{ Pre-intervention } & \multicolumn{2}{|c|}{ Post-intervention } & \multirow{2}{*}{$\begin{array}{l}\text { Pre vs. } \\
\text { Post } \\
\text { P-value }\end{array}$} \\
\hline & Mean \pm SD & P-value & Mean \pm SD & P-value & \\
\hline \multicolumn{6}{|l|}{ Gender } \\
\hline Male & $70.08 \pm 27.48 \%$ & 0.033 & $92.94 \pm 13.57 \%$ & 0.565 & $<0.001$ \\
\hline Female & $56.27 \pm 30.64 \%$ & & $91.3 \pm 15.72 \%$ & & $<0.001$ \\
\hline \multicolumn{6}{|l|}{ Education } \\
\hline Illiterate & $53.88 \pm 29.55 \%$ & 0.001 & $91.07 \pm 15.62 \%$ & 0.391 & $<0.001$ \\
\hline Literate & $74.11 \pm 26.09 \%$ & & $93.41 \pm 13.26 \%$ & & $<0.001$ \\
\hline \multicolumn{6}{|c|}{ Income (Pakistani Rupees) } \\
\hline$\leq 30,000$ & $63.72 \pm 28.37 \%$ & 0.484 & $93.77 \pm 12.00 \%$ & 0.155 & $<0.001$ \\
\hline$>30,000$ & $66.07 \pm 32.03 \%$ & & $88.75 \pm 18.73 \%$ & & $<0.001$ \\
\hline
\end{tabular}


Health care systems with limited resources that are more focused on medical interventions may not realize that without patient education complete benefit cannot be achieved. Our study demonstrates that a simple intervention based on a pictorial brochure and practical demonstration is sufficient to improve knowledge in all groups of patients including bringing illiterate patients with lower baseline knowledge at par with more educated patients.

A strength of our study is that it addresses patients from all socioeconomic groups and demonstrates an improvement across all groups. Moreover, the effects of the intervention could be observed even after a period of 10 days which demonstrated that its benefits lasted beyond short-term recall. Usage of pictorials also makes this tool universal across all languages.

\section{Limitations}

Limitations of our study include that many of the misconceptions may be rooted in local culture or myths and may not be applicable to other situations especially where patients are able to access knowledge on demand. Moreover, it is a single center study with its inherent limitations. There may also be a bias as the assessments were performed by members of the same team as provided the education. Bias may also have been introduced by the study design that excluded patients who did not appear in the follow-up. Moreover, as there was no control group improvement in knowledge in the intervening 10-day period from other sources cannot be ruled out.

\section{Conclusion}

Patients with pacemakers may have multiple misconceptions that can result in unnecessary restrictions. A simple educational intervention using a pictorial tool can successfully correct these. This study emphasizes the important role of counseling and patient education as part of holistic treatment of the disease.

\section{Abbreviations}

ERC: Ethics review committee; IQR: Inter quartile range; N.I.C.V.D.: National Institute of Cardiovascular Diseases; PPM: Permanent pacemaker; SD: Standard deviation; MRI: Magnet resonance imaging; CT: Computerized tomography; USD: United States dollar.

Acknowledgements

Not Applicable.

\section{Authors' contributions}

All authors have made substantial contributions to the conception, design of the work, analysis, interpretation of data, and drafted the work or substantively revised it. In addition, KK was responsible for acquiring all data. All authors have approved the submitted version and agree both to be personally accountable for the author's own contributions and ensured that there are no questions related to the accuracy or integrity of any part of the work. All authors read and aproved the final manuscript.

Funding

No funding was used for conducting this study.

\section{Availability of supporting data}

The datasets used and/or analyzed during the current study are available from the corresponding author on reasonable request.

\section{Declaration}

Ethical approval and Consent to participate.

This study was approved by the Ethics Review Committee of the National Institute of Cardiovascular Diseases, Karachi, Pakistan. Ref \#: ERC-44/2019. Written consent was obtained from all patients participating in the study.

\section{Consent for publication}

Consent for Publication of individual data: Not applicable.

\section{Competing of interests}

The authors declare that they have no competing interests.

Received: 11 November 2020 Accepted: 31 May 2021

Published online: 05 July 2021

\section{References}

1. Kusumoto FM, Schoenfeld MH, Barrett C, Edgerton JR, Ellenbogen KA, Gold MR, et al. ACC/AHA/HRS guideline on the evaluation and management of patients with bradycardia and cardiac conduction delay: executive summary: a report of the American college of cardiology/American heart association task force on clinical practice guidelines, and the heart rhythm society. Circulation. 2018;140(8):e333-81.

2. Torrington $\mathrm{M}$, Botha $\mathrm{J}$, Weymar HW. What do pacemaker recipients think of their implantations? An exploratory study. S Afr Med J. 1985:68(3):163-6.

3. Aqeel M, Shafquat A, Salahuddin N. Pacemaker patients' perception of unsafe activities: a survey. BMC Cardiovasc Disord. 2008;14(8):31.

4. Roser M, Ortiz-Ospina E. Our World in Data . 2016 [cited 2020 Oct 17]; https://ourworldindata.org/literacy

5. Chen HM, Chao YF. Change in quality of life in patients with permanent cardiac pacemakers: a six-month follow-up study. J Nurs Res. 2002;10(2):143-50.

6. Su SF, Wu MS. Arrhythmia perception and quality of life in bradyarrhythmia patients following permanent pacemaker implantation. Clin Nurs Res. 2021;30(2):183-92.

\section{Publisher's Note}

Springer Nature remains neutral with regard to jurisdictional claims in published maps and institutional affiliations. 